\title{
Comparative Monolayer Investigations of Surface Properties of Negatively Charged Glycosphingolipids from Vertebrates (Gangliosides) and Invertebrates (SGL-II, Lipid IV)
}

\author{
Heinz Beitinger," Florian Schifferer," Mutsumi Sugita,"** Shigeko Araki, ${ }^{* * * *}$ \\ Mei Satake, ${ }^{* * * *}$ Dietmar Möbius, ${ }^{* *}$ and Hinrich Rahmann*,1 \\ "Zoological Institute, University of Stuttgart-Hohenheim, D-7000 Stuttgart 70, F.R.G.; " Max-Planck-Institute for \\ Biophysical Chemistry, D-3400 Gottingen-Nikolausberg, F.R.G.; *** Department of Chemistry, Faculty of Liberal \\ Arts and Education, Shiga University, Ohtsu, Shiga 520; and "*" Department of Neurochemistry, Brain Research \\ Institute, Niigata University, Niigata, Niigata 951
}

Received for publication, November 30, 1988

The surface properties of four negatively charged glycosphingolipids from vertebrates, the sialo-glycosphingolipids (=gangliosides) $\mathrm{G}_{\mathrm{M} 1}, \mathrm{G}_{\mathrm{Dla}}, \mathrm{G}_{\mathrm{T} \mathrm{Tb}}$ and a sulfo-glycosphingolipid (= sulfatide), and of the two negatively charged glycosphingolipids from lower invertebrates, the glucurono-glycosphingolipid Lipid IV and the aminophosphono-glycosphingolipid SGL-II were investigated in monolayers at the air/water interface. The molecular peculiarities under investigation were surface pressure $(\pi)$ and surface potential $(\Delta V)$ which are described for Lipid IV and SGL-II for the first time. The surface pressure/area isotherms of all glycosphingolipids were typical of a liquid-expanded monolayer and, with the exception of SGL-II, exhibited a phase transition to a liquid-condensed state at surface pressures above $20 \mathrm{mN} / \mathrm{m}$. The surface potential $/$ molecular area data found for gangliosides in the closely packed state at $\pi=30 \mathrm{mN} / \mathrm{m}\left(\mathrm{G}_{\mathrm{M} 1}: \Delta V=-17 \mathrm{mV} ; \mathrm{G}_{\mathrm{Dla}}: \Delta V=-35\right.$ $\mathrm{mV} ; \mathrm{G}_{\mathrm{T} \mathrm{b}}: \Delta V=-39 \mathrm{mV}$ ) showed only a slight influence of the additional number of negatively charged residues. For Lipid IV, the surface behavior was very similar to $G_{M I}$ both possessing one negative group per molecule, whereas in SGL-II also the surface potential data $(\Delta V=+173 \mathrm{mV})$ were different compared with $G_{\text {DIa }}$ both possessing two negative groups per molecule. The addition of $\mathrm{Ca}^{2+}$ condensed the monolayers of all glycolipids and increased the potential in the direction to more positive values, but these findings were less effective in SGL-II films. On the basis of monolayer results presented here, in biological membranes of invertebrates especially Lipid IV might play a similar role as the ganglioside $\mathrm{G}_{\mathrm{M}}$ in vertebrate cells.

In the animal kingdom, besides sialic (=neuraminic) acid three other negatively charged sugar moieties have been developed to be linked to glycosphingolipids, i.e. sulphate ester, glucuronic acid, and aminoalkylphosphonic acid. With regard to phylogenetical aspects the animal kingdom is subdivided into the Protostomia (lower invertebrate like worms, arthropods,molluscs, etc.) and the Deuterostomia (echinoderms and vertebrates).

Sulpho-glycosphingolipids and sialo-glycosphingolipids ( = gangliosides) have been found only amongst the Deuterostomia (for Refs. compare 1 and 2). In the latter case this is due to the fact that in the Protostomia sialic acid is absent $(1,3-5)$. Until now, glucuronic acid containing glycosphingolipids have been isolated from both deuterostomian

\footnotetext{
${ }^{1}$ To whom correspondence should be addressed.

Abbreviations: DOPC, di-oleoyl-phosphatidylcholine; Cer, ceramide ( $N$-acylsphingoid); NeuAc, $N$-acetyl-neuraminic acid; Sulf, sulfatide, $\mathrm{Gal}\left(3-\mathrm{SO}_{1}\right.$-) $\beta$ 1-1Cer; $\mathrm{G}_{\mu 1}$, Gal $\beta$ 1-3GalNAc $\beta 1-4 \mathrm{Gal}$ (3$2 \alpha$ NeuAc) $\beta 1-4 \mathrm{Glc} \beta 1$-1Cer; $\mathrm{G}_{\mathrm{D1}}$. NeuAc $\alpha 2-3 \mathrm{Gal} \beta 1$-3GalNAc $\beta 1$. $4 \mathrm{Gal}(3-2 \alpha \mathrm{NeuAc}) \beta 1-4 \mathrm{Glc} \beta 1-1 \mathrm{Cer} ; \mathrm{G}_{\mathrm{Tl}}$, NeuAc $\alpha 2.3 \mathrm{Gal} \beta 1$. 3GalNAc $\beta 1-4 \mathrm{Gal}$ (3-2 $\alpha$ NeuAc8-2 $\alpha$ NeuAc)- $\beta 1$-4Glc $\beta 1$-1Cer; SGLII, skin glycolipid, 3-OMe Gal $\beta$ 1-3GalNAca 1-3[6'-O.(2aminoethylphosphonyl)Gal $\alpha$ 1-2] (2-aminoethylphosphonyl-6). Gal $\beta$ 1-4Glc $\beta$ 1-1Cer; Lipid IV, GlcA4Me $\beta$ 1-4(GalNAc3Me $\alpha$ 1-3) Fuc $\alpha$ 1-4GlcNAc $\beta$ 1-2Man $\alpha$ 1-3(Xyl $\beta$ 1-2) Man $\beta$ 1-4Glc $\beta 1-C e r$.
}

tissue (human peripheral nerves; 6) and protostomian tissue (spermatozoa of the fresh water mussle Hyriopsis schlegelii, and the flies Calliphora vicinia and Lucilia caesar; $1,7,8$ ). Finally aminophosphonic acid containing glycosphingolipids have been found in the marine snail Aplysia kurodai (9-11) and the snail shellfish $(12,13)$.

With regard to the sialo-glycosphingolipids, namely the gangliosides, a number of important cellular functions were already discovered, which can be summarized under the following headings: asymmetric distribution in cell membranes, fluidizing effect in membranes, cell-cell recognition and adhesion (e.g. fertilization), cell-substrate adhesion, contact inhibition of cell growth, modulatory function for cell membrane receptors, membrane ion pumps, and ion channels (14-22). Concerning activator functions for membrane bound enzyme (proteinkinases) especially $\mathrm{Ca}^{2+}$-ganglioside complexes of individual ganglioside species were found to be most effective $(23,24)$.

In contrast to the gangliosides, the possible functional role of the other acidic glycosphingolipids, mentioned above, is almost still unknown. Their involvement in the process of fertilization, early cell differentiation, and other cellular functions similar to those of glycosphingolipids is under discussion (7). 
Phospholipids are ubiquitous constituents of animal cell membranes forming the lipid matrix for the membranes structure. In addition, sulfatides, which are cerebrosides containing a sulphate group esterified to the sugar moiety and carrying one negative charge are other important membrane constituents, especially major components of myelin in the central nervous system. With regard to the specific role of the glycosphingolipids it is of essential interest to compare the physicochemical properties of these substances and the other representative lipids.

The importance of all these lipids as structural and functional components of biological membranes has stimulated numerous studies on the surface properties of purified individual compounds as well as their mutual interaction in definite mixtures. Such investigations have included the peculiarities of amphiphilic lipids to form monomolecular films at the air/water interface. Investigations on monolayers as artificial and simplified semimembrane system have been found to be very suitable (25-28) also for testing the influence of different environmental conditions on the physicochemical properties of those cell membrane molecules.

The formation of a monolayer can be measured as a change in surface pressure $(\pi)$ and surface potential $(\Delta V$; 29). By determination of the surface pressure it is possible to get characteristic information on molecular packing and orientation of molecules in a highly organized two dimensional layer at the air/water interface. The surface potential is proportional to the change of the normal component of the dipole density with respect to the pure, uncovered subphase surface (e.g. pure water, buffer solution). It should be noted here that the measured total surface potential includes both the potential of the diffuse counter ion layer and the dipole potential.

The properties of phospholipid, sulfatide, and gan. glioside monolayers as well as their interaction with metal ions in the subphase, especially $\mathrm{Ca}^{2+}$, have been under intensive study by various groups of investigators (25-27, $30-37)$. The work reported here is an extension of our previous studies $(27,36,37)$, employing several naturally occurring glycosphingolipids, with emphasis on the influ. ence of $\mathrm{Ca}^{2+}$ on the individual molecular properties. In order to get more information about the possible functional role of the various acidic glycosphingolipids in the animal kingdom, in this paper for the first time comparative data are presented concerning the surface pressure and surface potential values of differently composed glycosphingolipids containing sialic acid (gangliosides $G_{M 1}, G_{D 10}, G_{T 16}$ from vertebrates; compare Fig. 1a), glucuronic acid (Lipid IV from the mussle Hyriopsis; Fig. 1b), and aminoalkylphosphonic acid (SGL II from the snail Aplysia; Fig. Ic). These data are compared with those of phosphatidylcholine (DOPC) and sulfatide (Sulf) as less complex standards.

\section{MATERIALS AND METHODS}

The glucuronic acid containing glycosphingolipids Lipid IV was isolated from spermatozoa of fresh water bivalve Hyriopsis schlegelii (7). The diphosphonopentaosylceramide or aminoalkylphosphono-glycosphingolipid SGL-II was purified from the crude glycolipid fraction of the skin of Aplysia kurodai, a marine gastropod $(11,38)$. The individual gangliosides $\left(G_{M 1}, G_{D 1 a}, G_{T 1 b}\right)$ were a kind gift from
Fidia, Italy. Sulf and dioleoylphosphatidylcholine (DOPC) were obtained from Sigma, F.R.G.

Monolayers were formed on $5 \mathrm{mM}$ triethanolamine (TEA)/HCl-buffer ( $\mathrm{pH}$ 7.4) as subphase, in some cases additionally with $0.01 \mathrm{mM} \mathrm{CaCl}_{2}$. At this $\mathrm{pH}$, the sulfatide as well as the gangliosides are negatively charged $(32,39)$. Lipids were spread on the subphase as solutions in chloroform-methanol $(2: 1, \mathrm{v} / \mathrm{v})$ by means of a Hamilton syringe. After spreading, the solvent was allowed to evaporate and the film to stabilize for $5 \mathrm{~min}$. The values reported are the averages of at least two- to fivefold runs with separately prepared films. All experiments were performed at $20 \pm$ $0.5^{\circ} \mathrm{C}$.

The film balance assembly has been described elsewhere (29). Briefly it consisted of a rectangular Teflon trough with an internal area of $355 \mathrm{~cm}^{2}$ and a depth of $0.8 \mathrm{~cm}$. The monolayer was compressed by a movable Dynal barrier. A Wilhelmy balance with a $1.5 \mathrm{~cm}$ wide filter paper was used to record surface pressures as a function of surface area. The surface potential $(\Delta V)$ was measured by a vibrating plate condensor using a plate diameter of $15 \mathrm{~mm}$, a plate. water surface distance of about $1 \mathrm{~mm}$, and a frequency of about $400 \mathrm{~Hz}$. The accuracy was about $1 \mathrm{mV}$. The surface pressure $(\pi)$ and the surface potential $(\Delta V)$ were measured simultaneously during monolayer compression.

\section{RESULTS AND DISCUSSION}

The surface pressure/area curves on $5 \mathrm{mM} \mathrm{TEA} / \mathrm{HCl}(\mathrm{pH}$ 7.4) subphase, for a phospholipid (DOPC), sulfatide (Sulf), and the glycosphingolipids of increasing complexity are shown in Fig. 2 (a-g; solid lines). These isotherms are of the form typically shown by lipids in the liquid-expanded state. With the exception of DOPC this can probably be attributed to the presence of large polar head groups and especially negatively charged sugar moieties which are fully ionized on a subphase of $\mathrm{pH} 7.4$ (Ref. in 27) and give rise to an electrostatic repulsion between negative charges of adjacent molecules (26).

Unlike the glycosphingolipids, the molecular packing of DOPC in organized two-dimensional monolayer films and the characteristics at the air/water interface are determined by two unsaturated fatty acid chains in the hydro. phobic portion and a relatively small and zwitterionic polar head group (40). The configurational freedom of the highly fluid oleoyl chains is reasonable for the surface requirement of DOPC.

As shown in Fig. 2a, during monolayer compression, both the surface pressure and the surface potential of DOPC increase for areas smaller than $1.14 \mathrm{~nm}^{2}$ which can be correlated with the beginning of the removal of the hydrocarbon chains from the water surface ("lift off"). The limiting area is slightly less than $0.6 \mathrm{~nm}^{2} /$ molecule and the onset of film collapse is indicated at about $45 \mathrm{mN} / \mathrm{m}$. The aree and the surface pressure that are attributed to the maximum in surface potential $(\Delta V=+440 \mathrm{mV})$ can be interpreted as the molecular area of most close packing by an upright molecule and the surface pressure of film collapse. These data are in agreement with the literature (e.g. Ref. 41).

Unlike the phospholipids, the hydrophobic portion of the different glycosphingolipids consists of ceramide and is, on the average, very similar. The presence of successive 


\section{Deuterostomia}

\section{a. sialo-glycosphingolipid (vertebrates)}

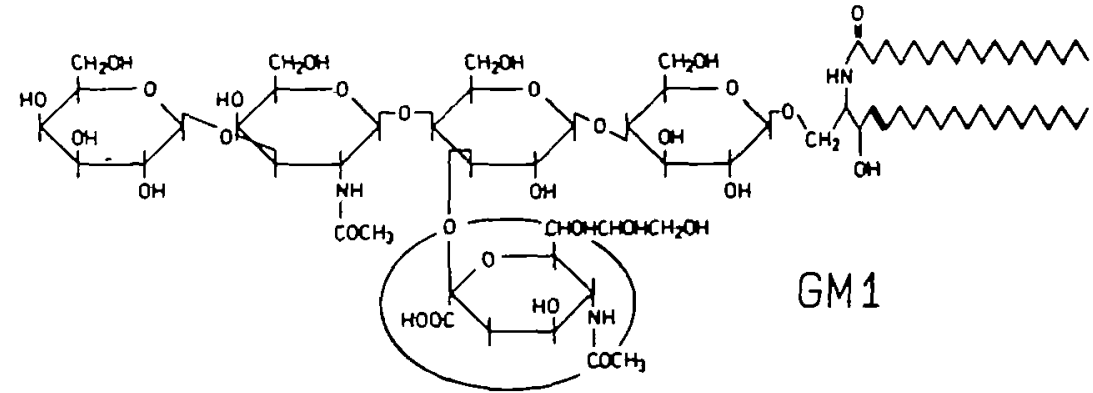

GalB1-3GaiNACB1-4Gal(3-2aNeUAC)B1-4GIcß1-1Cer

\section{Protostomia}

\section{b. glucurono-glycosphingolipld (mussle)}

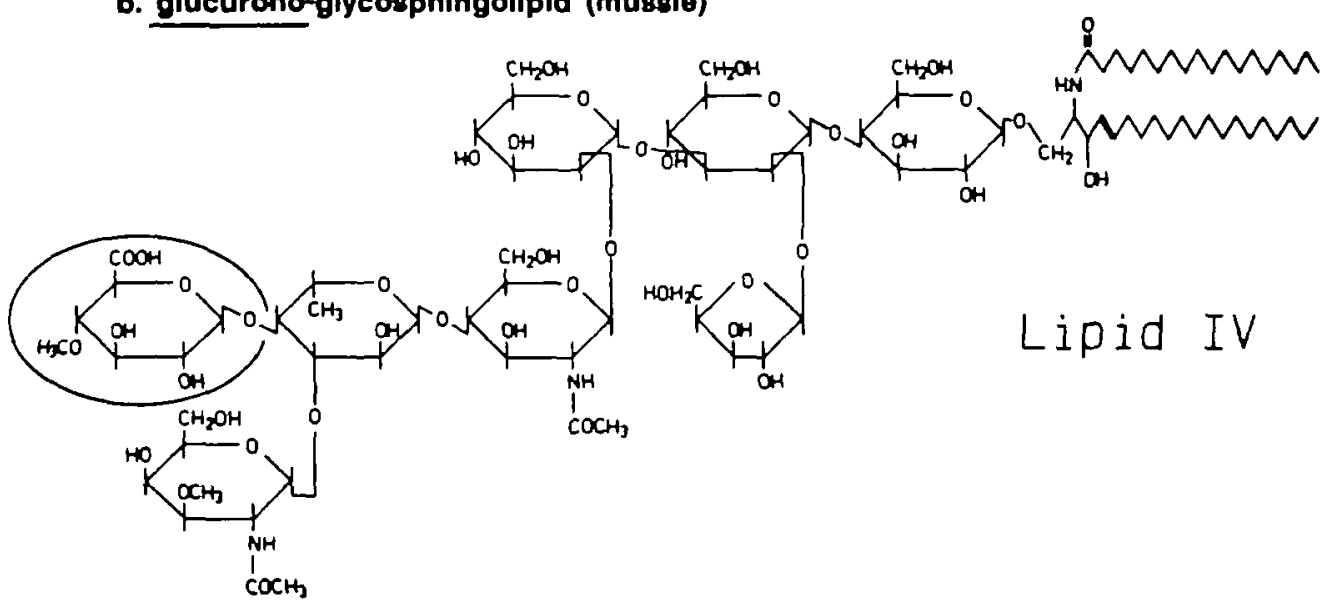

GlCA4Meß1-4(GolNAC3Mea1-3) Fuca 1-4GlcNAcB 1-2Mana1-3(XylB 1-2) ManB 1-4GlcB 1- 1Cer

\section{c. aminophosphono-glycosphingolipld (snali)}

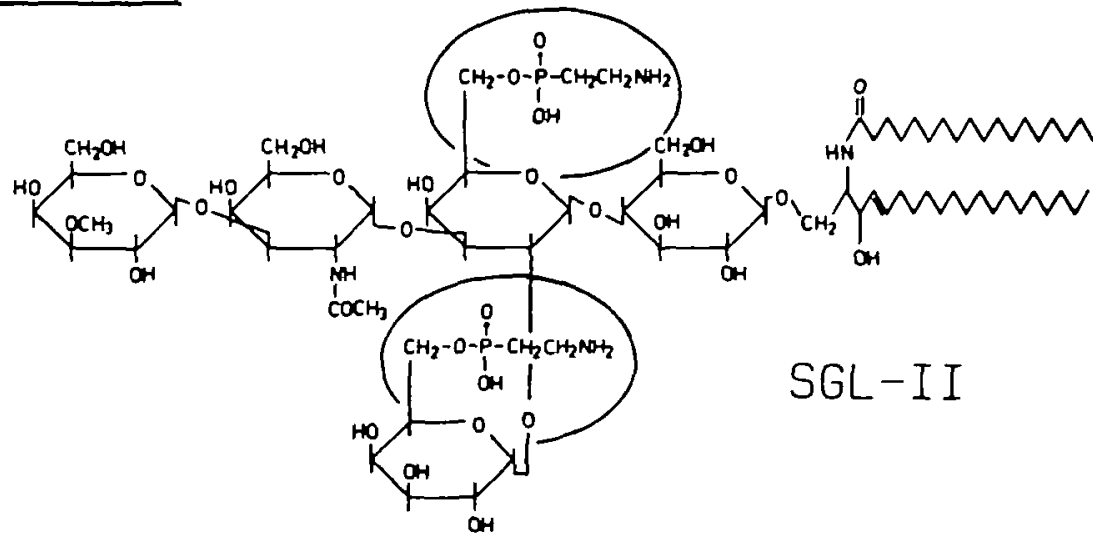

3-OMeGalB1-3GalNAca1-3[6'-O-(2-ominoethylphosphonyl)Gala1-2](2-aminoethylphosphonyl-6)Galn 1-4Gleß1-ICer

Fig. 1. Structures of three acldic glycosphingolipids used in this monolayer studies. (a) sialo-glycosphingolipid $G_{\mathrm{s}_{1}}$ (from deuterostomia), (b) glucurono-glycosphingolipid Lipid IV, and (c) aminophosphono-glycosphingolipid SGL-II (both from protostomia). 
charged or uncharged sugar residues in the large polar head group determines and modifies the molecular conformation, molecular packing, and electric potential at the air/ water interface (26). The mean molecular areas are increased due to the electrostatic repulsions by one or more negative charges and the hydrocarbon chains will have a considerable freedom to coil or tilt $(33,42)$. This leads to an increased compressibility and liquid character especially of ganglioside monolayers (33).

The complexity and negative charge of the polar head group of SGL-II and Lipid IV has also a profound influence on the molecular area and surface potential and should be comparable to gangliosides. For the first time, both surface pressure/area and surface potential/area isotherms are described for SGL-II and Lipid IV.

During compression of glycosphingolipid monolayer films, the surface pressure/area isotherms exhibit a shoulder (only weak for SGL-II), which can be interpreted as a transition between a liquid-expanded and a liquid-condensed phase (Ref. in 27). From sulfatide up to ganglioside $\mathrm{G}_{\mathrm{T} 1 \mathrm{~b}}$ (Fig. 2, b-e), the curves are shifted to higher values of area/molecule at a given surface pressure and the collapse pressures decreased due to the increased number of carbohydrate residues. This is in agreement with Maggio et al. (33), that the introduction of negatively charged sialyl residues causes a decrease of the collapse pressure. Further details are described in two recent papers $(36,37)$.

The surface pressure/area isotherm of Lipid IV (Fig. 2g) is closely related to $G_{M 1}$ (one negative charge) although its polar head group is larger. The isotherm of SGL-II (Fig. 2f) is strikingly different. The presence of two negative charges by aminoalkylphosphonic acids in the head group has an increasing effect on the liquid character with only a slight phase transition shoulder at high surface pressure (in the range of 30 to $40 \mathrm{mN} / \mathrm{m}$ ). In comparison to the gan. gliosides, especially $\mathrm{G}_{\mathrm{D} 1 \mathrm{a}}$, the molecular film is more stable with a collapse pressure of $55 \mathrm{mN} / \mathrm{m}$.

The surface potential $(\Delta V)$ of sulfatide carrying one negative charge is positive, about $\Delta V=+69 \mathrm{mV}$ in a closely packed state $(\pi=30 \mathrm{mN} / \mathrm{m})$. All gangliosides investigated here give rise to negative values, but only a slight influence of the additional number of sialic acid residues with respect to the potential values is given from $\mathrm{G}_{\mathrm{M} 1}$ up to $\mathrm{G}_{\mathrm{T} 1 \mathrm{~b}}$, for instance (at $\pi=30 \mathrm{mN} / \mathrm{m}$ ) $\mathrm{G}_{\mathrm{M}_{1}}$ : $\Delta V=-17 \mathrm{mV} ; \mathrm{G}_{\mathrm{D} 1 \mathrm{a}}: \Delta V=-35 \mathrm{mV} ; \mathrm{G}_{\mathrm{T} 1 \mathrm{~b}}: \Delta V=-39 \mathrm{mV}$. Figure 2, c-e, also indicates variations in surface potentials with decreasing surface area of the three monolayers in the phase transition region. As seen from these curves the surface potential increases slightly before the shoulder is reached in the $\pi$-curves, and after this, the surface potential shows a decrease up to the collapse of films.

The octaglycosylceramide Lipid IV (Fig. $2 \mathrm{~g}$ ) again exhibits a very similar behavior to the ganglioside $G_{M 1}$ (Fig. $2 c$ ), whereas SGL II (Fig. 2f) is characterized by only a slight phase transition shoulder in the $\pi$-curve, and a continuous increase in the $\Delta V$-isotherm (at $\pi=30 \mathrm{mN} / \mathrm{m}: \Delta V=$ $+173 \mathrm{mV}$ ).

The monofilm behavior at lateral surface pressures of 20 and $30 \mathrm{mN} / \mathrm{m}$ is of special interest, since these values are assumed to be those for natural membranes.

For all glycosphingolipids, the compression requires a very high energy input in each state of the monolayer films. The resistance of the system to compression increases because of the high activation energy involved when the large polar and charged headgroups are oriented in a monolayer of closely packed molecules.

As shown in Fig. 2 (a-g; interrupted lines), the addition of $\mathrm{Ca}^{2+}$ in the presence of a $5 \mathrm{mM}$ TEA/HCl $(\mathrm{pH} 7.4)$ buffered subphase has no effect on DOPC (Fig. 2a), but a pronounced effect on the properties of glycosphingolipid monolayers. For neutral phospholipid films it is indicated, that bivalent cations, especially $\mathrm{Ca}^{2+}$, do not exhibit an appreciable interaction (25). On the other hand there is a substantial interaction with monofilms of acidic phospholipids (25) and negatively charged glycosphingolipids $(26,27,36,37)$.

In general the main characteristics of the calcium addition to ganglioside monolayers are: (a) monolayer condensation as indicated by a decrease of the surface pressure $(\pi)$ at definite molecular areas; (b) higher film stability, significant rise of the collapse pressure; (c) shift of the phase transition shoulder to lower surface pressure values; (d) slight increase of surface potential $(\Delta V)$ in the direction of more positive values.

The comparison between Lipid IV and $G_{M 1}$ exhibits that at this stage the properties of the two films are not identical, and differ in two points: in Lipid IV calcium influences only weakly the phase transition shoulder as well as the stability of the film (collapse pressure). The curves for SGL II indicate only a weak deviation from the $\pi$-and $\Delta V$-curves without any calcium. It is of interest to note here that the calcium concentration is very low and effects might be observed in the presence of higher concentrations. In future experiments we design to investigate more intengively the influence of different mono- and divalent cations as well as the effect of ionic strength and $\mathrm{pH}$.

Data presented in this paper emphasize several physicochemical aspects especially with respect to some relevant sialic acid-, glucuronic acid-, and aminophosphonic acidcontaining glycosphingolipids. Generally, when oriented at the air/water interface, and under high lateral pressure (e.g. $\pi=30 \mathrm{mN} / \mathrm{m}$ ), the molecules build up an asymmetric monolayer of closely packed methyl groups of the hydrocarbon chains at the monolayer/air interface, and of relatively closely packed and hydrated polar head groups at the monolayer/water interface. The carbohydrate residues of the polar head group contribute with an electrical field in the opposite direction to that of the hydrocarbon chains (26), here especially the terminal $\mathrm{CH}_{3}$-groups (43). Since the dipole potential contribution of the terminal $\mathrm{CH}_{3}$ groups in closely packed lipid monolayers is constant (43), measurable changes in the total surface potential $(\Delta V)$ depend on variations in the so-called head group region potential, that includes hydrations effects as well as, in ionized monolayers, the potential of the diffuse counter ion layer.

Based on this knowledge, it will be concluded in future experiment, how much quantitatively the contribution of the polar headgroup is to the total surface potential. With respect to the different glycosphingolipids investigated this is of essential interest, because, in biological bilayer systems, the effective dipole strength and the effective surface potential of the hydrated polar groups determines the electrostatic properties of bilayer surfaces.

The behavior of these compounds tested in a model membrane system like monolayers, for instance their 

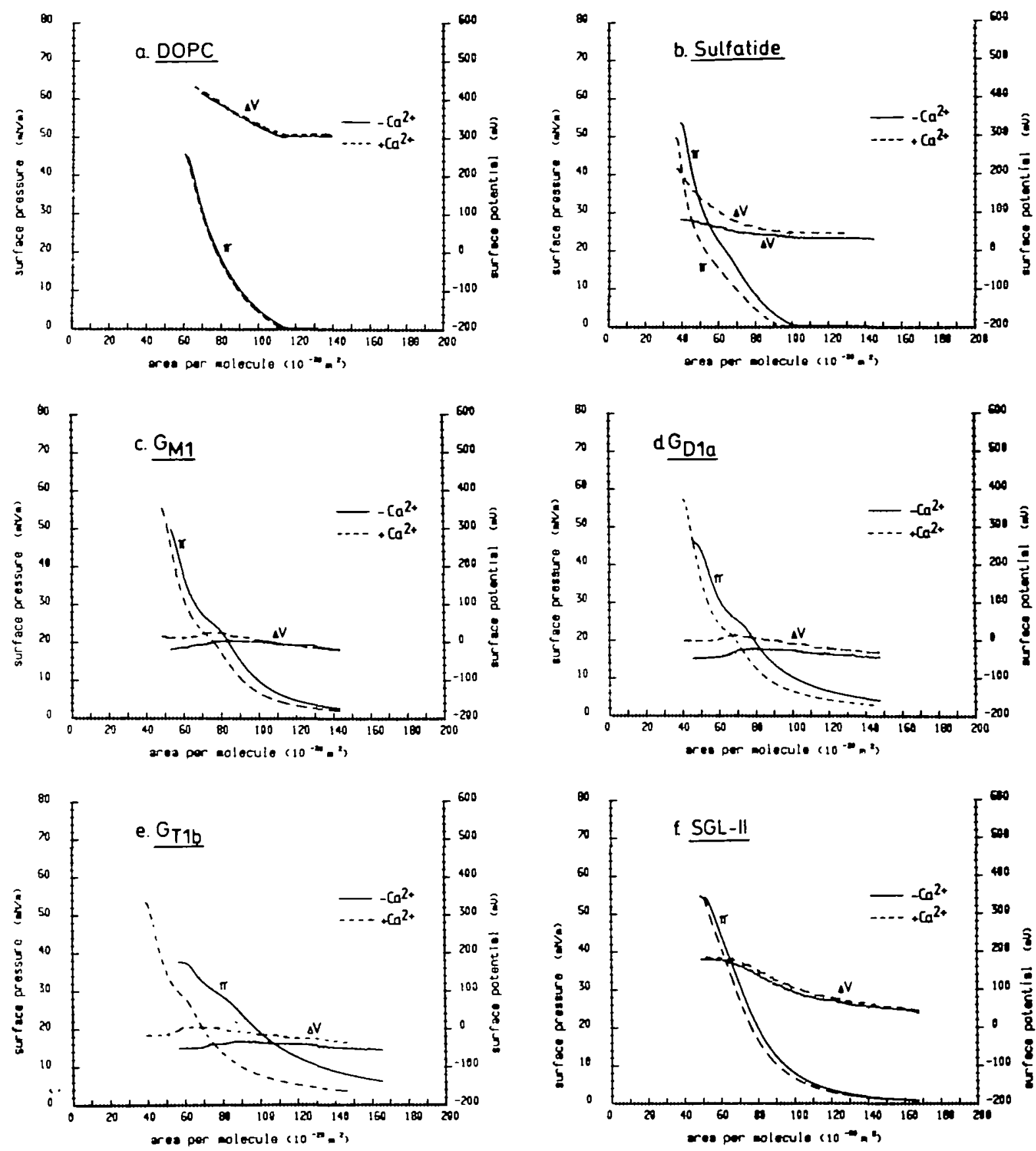

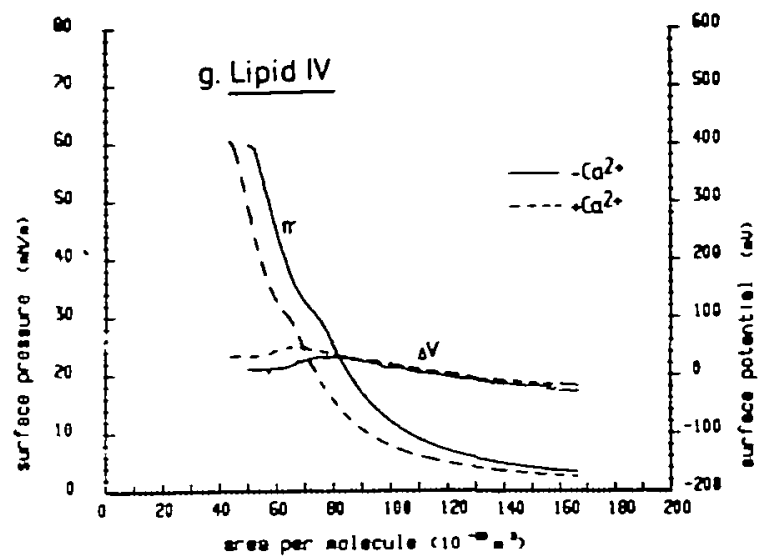

Fig. 2. Surface pressure $(\pi)$ /and surface potential $(\Delta V)$ /area isotherms, respectively, of (a) DOPC, (b) Sulf, (c) $G_{M 1}$, (d) $G_{D I a,}$, (e) $G_{T 1 b}$, (f) SGL-II, (g) Lipid IV. Subphase: $5 \mathrm{mM}$ TEA/HCl buffer (pH 7.4) with (- -)

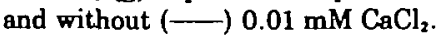


interaction with divalent cations like calcium, and, on the other hand, their presence in specific biological membranes of vertebrates or invertebrates point out the relevance of such investigations to functional problems of biology.

\section{REFERENCES}

1. Dennis, R.D., Geyer, R., Egge, H., Peter-Katalinic, J., Keller M., Menges, H., \& Wiegandt, H. (1987) in Gangliosides and Modulation of Neuronal Functions (Rahmann, H., ed.) NATO ASI Series Vol. 7, pp. 351-358, Springer Verlag, Berlin, Heidelberg, New York, Tokyo

2. Hilbig, R. \& Rahmann, H. (1987) in Gangliosides and Modulation of Neuronal Functions (Rahmann, H., ed.) NATO ASI Series Vol. 7, pp. 333-350, Springer Verlag, Berlin, Heidelberg, New York, Tokyo

3. Segler,, K., Rahmann, H., \& Rb̋aner, H. (1978) Biochem. Syst Ecol 6, 87-93

4. Svennerholm, L. (1970) in Handbook of Neurochemistry (Lajtha, A., ed.) Vol. 3, pp. 426, Plenum Press, New York

5. Komai, Y., Matsukawa, S., \& Satake, M. (1971) J. Biochem. 70, 367-369

6. Chou, K.H., Ilyas, A.A., Evans, J.E., Quarles, R.H., \& Jungalwala, F.B. (1985) Biochem. Biophys. Res. Commun. 128, 383388

7. Hori, T., Sugita, M., Ando, S., Tsukada, K., Shiota, K., Tsuzuki, M., \& Itasaka, O. (1983) J. Biol. Chem. 258, 2239-2245

8. Sugita, M., Itonori, S., Inagaki, F., Itasaka, O., \& Hori, T. (1988) Prac. Jpn. Conf. Biochem. Lipids 30, 77-80

9. Araki, S. \& Satake, M. (1981) Neurosci. Lett. 22, 179-182

10. Araki, S. \& Satake, M. (1985) Biochem. Int. 10, 603-610

11. Araki, S., Satake, M., Ando, S., Hayashi, A., \& Fujii, N. (1986) J. Biol. Chem. 261, 5138-5144

12. Hayashi, A. \& Matsuura, F. (1971) Biochim. Biophys. Acta 248, $133-136$

13. Matsubara, T. \& Hayeshi, A. (1986) Proc. Jpn. Conf. Biochem. Lipids 28, 33-36

14. Ledeen, R.W. (1978) J. Supramol. Struct. 8, 1-17

15. Hakomori, S.-I. (1981) Annu. Rev. Biochem. 50, 733-764

16. Leon, A., Facci, L., Benvengnu, D., \& Toffano, G. (1982) Dev. Neurosci. 5, 108

17. Tettamanti, G., Sonnino, S., Ghidoni, R., Masserini, M., \& Venerando, B. (1985) in Physics of Amphiphiles: Micelles, Vesicles and Microemulsions (Corso, X.C., ed.) pp. 607-636

18. Bremer, E.G., Schlessinger, J., \& Hakomori (1986) J. Biol. Chem. 261, 2434-2440

19. Gore, P.J., Singh, S.P., \& Brooks, D.E. (1986) Biochim. Biophys.
Acta 876, 36-47

20. Spiegel, S., Handler, J.S., \& Fishman, P.H. (1986) J. Biol. Chem 261, 15755-15760

21. Rahmann, H. (1987) in Gangliosides and Modulation of Neuronal Functions (Rahmann, H., ed.) NATO ASI Series Vol. 7, pp. 501-521, Springer Verlag, Berlin, Heidelberg, New York, Tokyo

22. Nagata, Y., Ando, M., Iwata, M., Hara, A., \& Taketomi, T. (1987) J. Neurochem. 49, 201-207

23. Goldenring, J.R., Otis, L.C., Yu, R.K., \& DeLorenzo, R.J. (1985) $J$. Neurochem. 44, 1229-1234

24. Tsuji, S., Nakajima, J., Sasaki, T., \& Nagai, Y. (1985) J. Biochem 97, 969-972

25. Papahadjopoulo8, D. (1968) Biochim. Biophys. Acta 163, 240254

26. Maggio, B., Cumar, F.A., \& Caputto, R. (1981) Biochim. Biophys. Acta 650, 69-87

27. Probst, W., M8bius, D., \& Rahmann, H. (1984) Cell. Mol. Neurobiol. 4, 157-176

28. Probst, W. Rahmann, H. (1987) in Gangliosides and Modulation of Neuronal Functions (Rahmann, H., ed.) NATO ASI Series Vol. 7, pp. 139-154, Springer Verlag, Berlin, Heidelberg, New York, Tokyo

29. Vogel, V. \& Mrbius, D. (1985) Thin Solid Films 132, 205-219

30. Rojas, E. \& Tobias, J.M. (1965) Biochim. Biophys. Acta 84, 394404

31. Phillips, M.C. \& Chapman, D. (1968) Biochim. Biophys. Acto 163, 301-313

32. Quinn, P.J. \& Sherman, W.R. (1971) Biochim. Biophys. Acto 233, 734-752

33. Maggio, B., Cumar, F.A., \& Caputto, R. (1978) Biochem. J. 171 , $559-565$

34. Maggio, B., Cumar, F.A., \& Caputto, R. (1980) Biochim. Biophys. Acto 189, 435-440

35. Fidelio, G.D., Maggio, B., \& Cumar, F.A. (1986) Biochim Biophys. Acta 854, 231-239

36. Beitinger, H., Probst, W., Möbius, D., \& Rahmann, H. (1987) $J$ Biochem. 102, 963-966

37. Schifferer, F., Beitinger, H., Rahmann, H., \& Mobius, D. (1988) FEBS Lett. 233, 158-162

38. Abe, S., Araki, S., \& Satake, M. (1986) Biomed. Res. 7, 47-51

39. Abramson, M.B., Yu, R.K., \& Zaby, V. (1972) Biochim. Biophys. Acta 280, 365-372

40. Phillips, M.C., Ladbrooke, B.D., \& Chapman, D. (1970) Biochim. Biophys. Acta 186, 35-44

41. Paltauf, F., Hauser, H., \& Phillips, M.C. (1971) Biochim. Biophys. Acta 249, 539-547

42. Seelig, A. \& Seelig, J. (1974) Biochemistry 13, 4839-4845

43. Vogel, V. \& Morbius D. (1988) Thin Solid Films 159, 73-81 